\title{
Investigation of some mechanisms for formation of exciton absorption bands in layered semiconductor $n$-InSe and p-GaSe crystals
}

\author{
Yu.I. Zhirko, I.P. Zharkov \\ Institute of Physics, NAS of Ukraine, 46 Prospect Nauki, 03650 Kyiv-39, Ukraine \\ Phone: +38(044)265 1464; e-mail: zhirko@nas.gov.ua
}

\begin{abstract}
We show that processes of creation, radiation and decay of the ground $(n=1)$ and excited exciton states in layered $n$-InSe and $p$-GaSe crystals involve direct (photon $\rightarrow$ exciton $\rightarrow$ photon, at $k=0$ ), as well as indirect vertical (photon \pm phonon $\rightarrow$ exciton $\rightarrow$ photon \pm phonon, at $k \cong 0$ ), optical transitions. For the $n=1$ exciton state both transitions are compatible. For the excited exciton states the above transitions are not compatible; as a result, the integral intensity of absorption bands for excited exciton states, $K_{n}$, exceeds $K^{0} / n^{3}$ (where $K^{0}$ is the classic value for the $n=1$ exciton absorption band) and grows with temperature. It is shown that presence of two-dimensional gas of charge carriers (electrons/holes localized in quantum wells) that are degenerate with excitons in the momentum space leads to suppression of the oscillator strength of exciton transition for ground, as well as excited, states. It was found experimentally that growth of temperature in $p$-GaSe crystals results in holes redistribution to the higher-energy states. This appears as consecutive (from the ground to excited states) suppression and re-establishment of the integral characteristics of exciton absorption bands.
\end{abstract}

Keywords: exciton absorption, layered crystal, $n$-InSe, $p$-GaSe, quantum well.

Paper received 24.12.02; accepted for publication 16.06.03.

\section{Introduction}

Layered crystals InSe and GaSe belong to the III-VI semiconductor compounds. They are characterized by weak van der Waals bonding between the layers and strong ionic-covalent intralayer bonding. Their crystal layers involve four atomic planes (-Se-In, Ga-In, Ga-Se-); arrangement of atoms within a layer corresponds to the $D_{3 h}^{1}$ space group. On the whole, strong anisotropy of chemical bonding does not lead to substantial peculiarities in behavior of excitons in the above crystals, as compared to other binary semiconductors. The Wannier-Mott excitons in III-VI compounds are three-dimensional (3D), as in the well-studied II-VI and III-V compounds. However, the lower symmetry of layered crystals results in higher values of the electron-phonon deformation potential. Therefore, excitons and electrons (holes) are scattered by homopolar optical phonons. Contrary to electron conductivity ( $n$-InSe), hole conductivity ( $p$-GaSe) in layered crystals is highly anisotropic: the values of hole effective mass along the layers and normal to them differ by a factor of four to five.
It should be also noted that the direct (D) optical transitions in InSe and GaSe are allowed but slightly. So the oscillator strengths for exciton and interband transitions are actually less than those in the II-VI crystals by a factor of 20-60. Due to chemical bonding anisotropy in layered crystals, a possibility exists for impurity insertion into the layers (by doping during growth), as well as into the interlayer space (by intercalation). Some authors believe that substantial ( $>0.1 \%$ by weight) amount of dopant results in appearance of planar impurity aggregates. They are deposited at stacking faults and lead to appearance of $2 \mathrm{D}$ electron subbands in $n$-type crystals.

All the above features, together with the worked-out growth technology and easiness of sample preparation, make excellent possibilities for experimental investigation of the exciton absorption spectra not only in 3D semiconductor crystals, but also in crystal structures with 2D quantum wells (QWs) filled with charge carriers.

At the same time, these crystals (InSe), due to considerable volume of their unit cell, its mass and bandgap, can serve for production of solar cells, IR converters and (at proper intercalation) chargeable cells as well. 
Yu.I. Zhirko, I.P. Zharkov: Investigation of some mechanisms for formation of ...

\section{Experimental results and discussion}

In our previous papers $[1,2]$ we have made temperature investigations of exciton absorption spectra for Bridgmangrown $n-\operatorname{InSe}(T=4.5-100 \mathrm{~K})$ and $p-\operatorname{GaSe}(T=4.5-300 \mathrm{~K})$ single crystals - pure, doped and intercalated with irongroup impurities. In particular, eight samples of InSe crystals of different thicknesses were studied in [1] - both pure and doped with iron-group impurities $(\mathrm{Cr}, \mathrm{Ni}$ and $\mathrm{Mn}$ ), the impurity concentrations being up to $0.1 \%$ by weight. In [2] we investigated GaSe crystals pure, doped with $\operatorname{Mn}(0.1 \%$ by weight $)$ and intercalated with $\operatorname{Mn}(0.1 \%$ by weight). The concentration of uncontrollable contaminations was below $10^{16} \mathrm{~cm}^{-3}$.

The calculations of exciton absorption spectra in InSe and GaSe crystals made within the framework of the traditional Elliott-Toyozawa theory have shown that growth of the integral intensity $K_{1}$ of the $n=1$ exciton absorption band is due to polariton mechanism. It is qualitatively described with the quantum-statistical theory of light propagation in crystals advanced by Davydov and Serikov [3]. For these crystals the following simple analytic expression has been found in $[1,2]$ for $K_{1}$ dependence on the band half-width $\Gamma_{1}$ :

$$
K_{1}\left(\Gamma_{1}\right)=K^{0} \times \frac{\sqrt{\Gamma_{1}^{2}-2 \Gamma_{1}^{\prime 2}(0)}}{\Gamma_{1}},
$$

where $K^{0}$ is the classic value of the integral intensity of the $n=1$ exciton absorption band. The half-width at halfmaximum, $\Gamma_{1}$, for the $n=1$ exciton absorption band is associated with the lifetime $\tau=\hbar / 2 \Gamma_{1}$ and depends on both homogeneous and inhomogeneous broadening:

$$
\begin{aligned}
& \Gamma_{1}^{2}(T)=\Gamma_{1}^{\prime 2}(T)+\Gamma_{\text {inh }}^{2}= \\
& =\Gamma_{1}^{\prime 2}(0) \cdot\left[\Theta+\beta_{1} n^{*}(T)\right]^{2}+\Gamma_{\mathrm{inh}}^{2} .
\end{aligned}
$$

Here $\Gamma_{\text {inh }}$ is the additive inhomogeneous component of the exciton absorption band. It does not depend on temperature and is due to exciton scattering on the crystal lattice defects. $\Gamma_{1}^{\prime}(T)$ is the homogeneous component. It increases with crystal temperature and is due to exciton scattering by phonons. $\Gamma_{1}^{\prime}(0)=g^{2}\left[\hbar \Omega\left(R_{0}-\hbar \Omega\right)\right]^{1 / 2}$, where $g$ is the exciton-phonon interaction constant. $n^{*}(T)=$ $=\left[\exp \left(\hbar \Omega / k_{\mathrm{B}} T\right)-1\right]^{-1} ; k_{\mathrm{B}}$ is the Boltzmann constant; $\beta_{1}=$ $=1+\left[\left(R_{0}+\hbar \Omega\right) /\left(R_{0}-\hbar \Omega\right)\right]^{1 / 2} ; R_{0}$ is the exciton binding energy; $\hbar \Omega$ is the energy of the optical phonon at which exciton is being scattered, $\Theta=1$ at $R_{0}<\hbar \Omega$ and $\sqrt{2}$ at $R_{0}>\hbar \Omega$.

Fig. 1a presents the experimentally determined [1] $K_{1}\left(\Gamma_{1}\right)$ dependencies for InSe crystal when $\Gamma_{1}$ broadening is due to temperature increase (triangles) or defect number growth (squares). Triangles in Fig. 1c show the experimental $K_{1}(T)$ dependence for a high-quality InSe crystal (with small inhomogeneous broadening).

Curve 2 (see Figs 1a, c) is plotted according to expression (1) for $\Gamma_{\min }=\sqrt{2} \Gamma_{1}^{\prime}(0)=1.84 \mathrm{meV}$ and $\hbar \Omega=$
$=13.6 \mathrm{meV}$. It is in satisfactory agreement with the model [3] and experimental $K_{1}\left(\Gamma_{1}\right)$ and $K_{1}(T)$ values. Curve 1 corresponds to the classic absorption, when $K_{1}=K^{0}$ is const. It should be noted that in InSe $K_{1}$ begins to grow at $\Gamma_{\min }=\sqrt{2} \Gamma_{1}^{\prime}(0)$, where $\Gamma_{1}^{\prime}(0)$ is determined from the experimental values of the temperature broadening of the $n=1$ exciton absorption band. This growth occurs simultaneously with broadening the exciton absorption band. One should also note that the fact of $K_{1}$ growth with temperature is well known for the III-VI layered crystals [4-6], where it was associated, as a rule, with both the effect of polariton mechanism for exciton decay and role of indirect vertical (IV) optical transitions. The IV transitions are traditionally considered [7] to be involved in formation of exciton absorption bands at higher temperatures, when the polariton mechanism has worked yet. We believe, however, that the IV transitions can take an active part in exciton absorption band formation (broadening and increase of integral intensity) even at low temperatures.

\subsection{On indirect vertical transitions}

In [8], by the example of InSe crystal, an additional increase with crystal temperature has been found for the light absorption coefficient $\alpha(\hbar \omega)$ in the absorption region of excited $n=2,3, \ldots$ exciton states. It should be noted that such an increase of the integral intensity $K_{n}$ for the excited exciton states contradicts with the traditional theory of exciton absorption. According to this theory, at low concentrations of exciton gas, excitons that are in excited states are either scattered at defects or phonons (and, as a result, are absorbed), or relax to the ground state from which radiative (photons), as well as nonradiative (absorption) transitions occur. The condition $K_{n}=K_{0} / n^{3}=$ const has to hold in this case. For InSe and GaSe crystals, however, it was found that $K_{n}>K_{0} / n^{3}$ even at $T=4.5 \mathrm{~K}$, and the integral intensity $K_{n}$ growth continues with the crystal temperature.

To numerically calculate the contribution from excited states into the exciton absorption spectrum of GaSe, we assumed (as it was done in [8] for InSe crystals) that the character of exciton-phonon interaction does not change when going from the ground to excited exciton states. In this approximation (according to [8]), we applied for GaSe crystals the following generalized expression for exciton scattering from excited states by optical phonons:

$$
\begin{aligned}
& \Gamma_{n}^{2}(T)=\Gamma_{n}^{\prime 2}(T)+\Gamma_{\mathrm{inh}}^{2}= \\
& =\left[\Gamma_{n}^{\prime}(0) \cdot\left(1+\beta_{n}^{\prime} \cdot n^{*}(T)\right)\right]^{2}+\Gamma_{\mathrm{inh}}^{2},
\end{aligned}
$$

where $\mathrm{G}_{n}^{\prime}(0)=g^{2}\left[\hbar \Omega\left(R_{0} / n^{2}-\hbar \Omega\right)\right]^{1 / 2}, \beta_{n}^{\prime}=\sqrt{2}\left[\left(\beta_{n}{ }^{2}-1\right) / \beta_{n}\right]$, and $\beta_{n}=1+\left[\left(R_{0} / n^{2}+\hbar \Omega\right) /\left(R_{0} / n^{2}-\hbar \Omega\right)\right]^{1 / 2}$. It was shown (just as in the case of InSe) that the relation $\Gamma_{n}=\Gamma_{c}-$ $-\left(\Gamma_{c}-\Gamma_{1}\right) / n^{2}$ for the half-widths of the ground $\left(\Gamma_{1}\right)$, excited $\left(\Gamma_{n}\right)$ and continuum $\left(\Gamma_{c}\right)$ exciton states, which was experimentally obtained in [9] for sufficiently perfect 
Yu.I. Zhirko, I.P. Zharkov: Investigation of some mechanisms for formation of ...
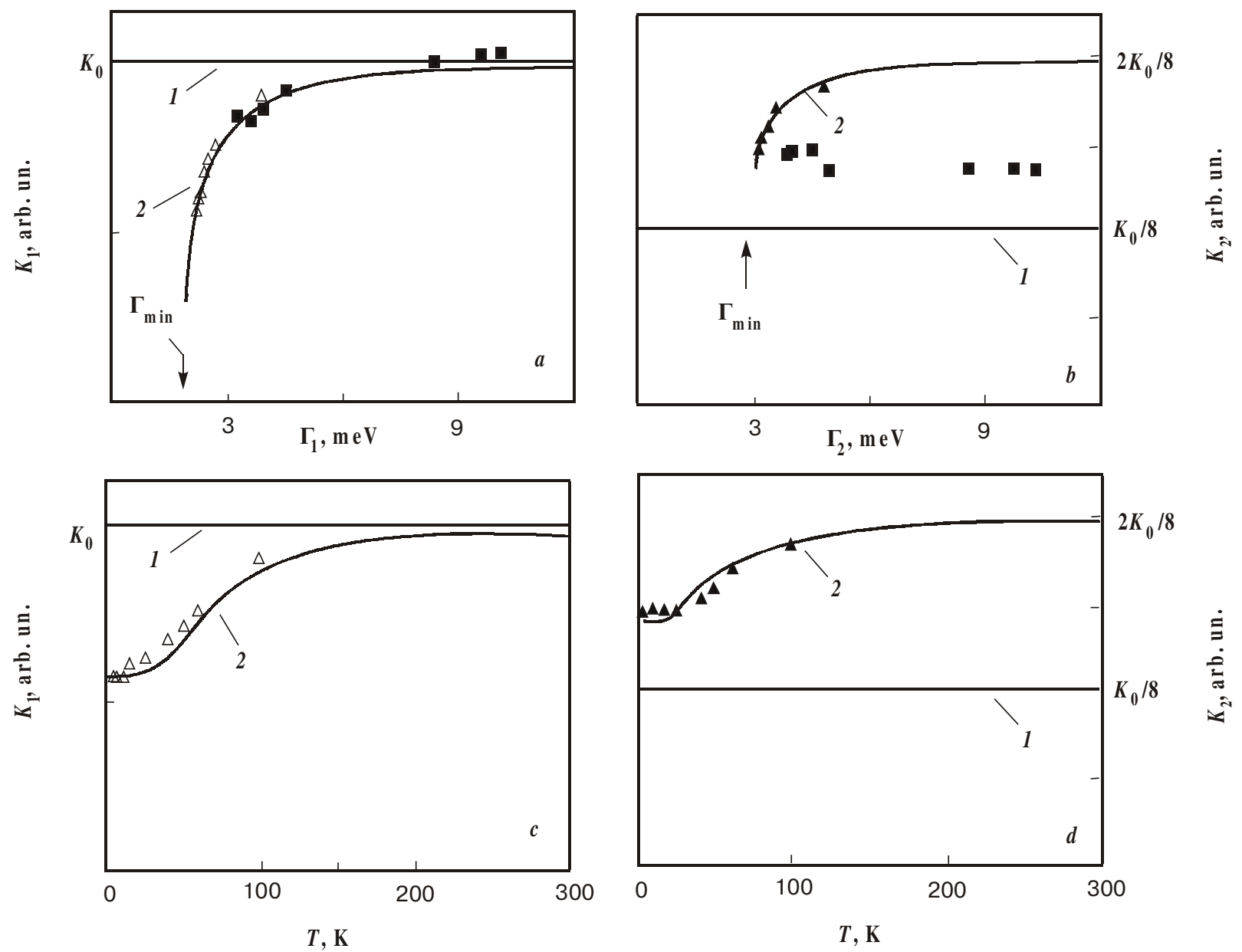

Fig. 1. $a, c-K_{1}\left(\Gamma_{1}\right)$ and $K_{1}(T)$ values for the $n=1$ exciton state in $n$-InSe crystal: samples $a$ ) $-h$ ) at $T=4.5 \mathrm{~K}$ (squares) and sample $a$ ) at temperatures from 4.5 up to $100 \mathrm{~K}$ (triangles). Curves 1 are plotted for classic absorption when $K_{1}\left(\Gamma_{1}\right)=K^{0} \equiv$ const. Curves 2 are plotted according to expression (1) with $\Gamma_{1}(T)$ according to expression (2). $b, d-K_{2}\left(\Gamma_{2}\right)$ and $K_{2}(T)$ values for the $n=2$ exciton state in $n$-InSe crystal: samples $a$ ) $-h$ ) at $T=4.5 \mathrm{~K}$ (squares) and sample $a$ ) at temperatures from 4.5 up to $100 \mathrm{~K}$ (triangles). Curves $l$ are plotted for classic absorption when $K_{2}\left(\Gamma_{2}\right)=K^{0} / 8$. Curves 2 are plotted according to expressions (5) at $\Gamma_{\text {inh }}=1.3$ meV with $\Gamma_{2}(T)$ according to expression (3).

GaSe crystals at $T=4.2 \mathrm{~K}$, may be expressed in the more accurate form:

$\Gamma_{n}^{\prime}(0)=\Gamma_{c}^{\prime}(0)-\frac{\Gamma_{c}^{\prime}(0)-\Gamma_{1}^{\prime}(0)}{n^{2}}$,

where $\Gamma_{1}^{\prime}(0), \Gamma_{n}^{\prime}(0)$ and $\Gamma_{c}^{\prime}(0)$ are the homogeneous components of the half-widths for the ground, excited and continuum exciton states, respectively, at $T=0$.

Using expression (3) and experimentally found in [1] $\Gamma_{1}(T)$ and $\Gamma_{\text {inh }}$ values for InSe, we obtained in [8] the values of half-widths $\Gamma_{n}$ for absorption bands of excited exciton states. Besides, we calculated the absorption spectra with allowance made for the ground and three first excited states, as well as (see [1]) the interband transition, absorption band with low concentration of shallow $(17 \mathrm{meV}$ ) donors, a shallow ( $45 \mathrm{meV}$ below the conduction band bottom) defect level, and the trap level $(130 \mathrm{meV}$ above the valence band top). Similar calculations (ground and three excited exciton states, interband transition and shallow acceptor level located on $20-30 \mathrm{meV}$ above the valence band) are made in this paper for GaSe crystals. The results obtained are discussed below.

Shown in Fig. $1 \mathrm{~b}$ are the values of $K_{2}$ (integral intensity of the $n=2$ exciton absorption band for InSe crystals) obtained for the cases when band broadening is due to increase of temperature (triangles) or defect number (squares). Fig. 1d presents the $K_{2}(T)$ dependence (triangles) for high-quality InSe crystals with a small number of defects resulting in insignificant inhomogeneous band broadening.

The straight line 1 demonstrates the case of classic absorption when $K_{2}=K^{0} / 2^{3}=$ const. One can see that temperature growth (triangles) leads to $K_{2}$ increase to values substantially over the classic ones. In the case when the number of defects grows (squares) $K_{2}=$ const, and 


\section{Yu.I. Zhirko, I.P. Zharkov: Investigation of some mechanisms for formation of ...}

increase of the half-width $\mathrm{G}_{2}$ is accompanied with drop of intensity in the peak of the exciton absorption band. Similar behavior has been found also for the $n=3$ exciton absorption band.

Prima facie, $K_{n}$ value increasing with temperature conflicts with the traditional theory of light absorption by excitons. This theory assumes (in accordance with the linear response theory) that $K_{n}=K^{0} / n^{3}$ is const, and broadening of an absorption band leads to decrease of its peak height. Nevertheless, the character of $K_{n}(T)$ dependence can be explained, if one takes into account the IV optical transitions. According to $[10,11]$, they also result in growth of the integral intensity of exciton absorption band. Contrary to the case of D transitions (photon $\rightarrow$ exciton $\rightarrow$ photon, at $k=0$ ), IV transitions (photon \pm phonon $\rightarrow$ exciton $\rightarrow$ photon \pm phonon, at $k \cong 0$ ) involve a phonon.

Fig. 2 shows a diagram of an IV transition via a virtual state in the crystal bandgap, with optical phonon creation (arrow 2) and annihilation (arrow 3). A D transition is shown with the arrow 1 .

In [8] it was shown that, for the ground exciton state at low concentrations of exciton gas, D and IV transitions are compatible. This means that, whatever the way of photon transition to exciton state, the reverse transition of exciton to photon state can proceed via $\mathrm{D}$, as well as IV, transition. It was also shown in [8] that phonons of the same energy $\hbar \Omega$ take part in both IV transition (process of exciton creation with further photon emission) and exciton scattering resulting in homogeneous broadening of the ground exciton state. In particular, for InSe and GaSe crystals such a phonon is a homopolar phonon of $A^{\prime}{ }_{1}$ symmetry $\left(\hbar \Omega_{\mathrm{InSe}}=13.6 \mathrm{meV}, \hbar \Omega_{\mathrm{GaSe}}=16.8 \mathrm{meV}\right)$.

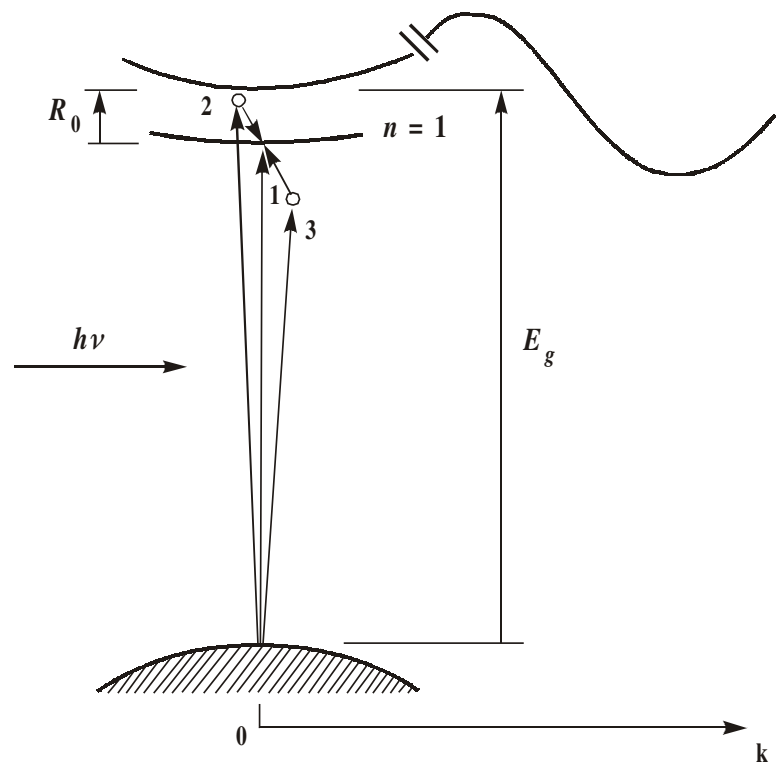

Fig. 2. Diagram of an indirect vertical transition via a virtual state in the crystal bandgap, with optical phonon creation (arrow 2) and annihilation (arrow 3). A direct transition is shown with arrow 1 .
Its energy is very close to the exciton binding energy $R_{0}$ in $\operatorname{InSe}\left(R_{0}=14.5 \mathrm{meV}\right)$ and $\operatorname{GaSe}\left(R_{0}=20.0 \mathrm{meV}\right)$ crystals.

Since $R_{0}>\hbar \Omega$ for InSe and GaSe crystals, then IV transitions are symmetric in them, i.e., they occur with optical phonon creation/annihilation. In this case, according to [8], the minimal half-width for the $n=1$ exciton absorption band, with allowance made for D and IV transitions, is $\Gamma_{\min }=\sqrt{2} \Gamma_{1}^{\prime}(0)$. Indeed, as it was shown in [1, 2], broadening of the $n=1$ exciton absorption bands in InSe and $\mathrm{GaSe}$, according to expression (2) and experimental results, begins at $\Gamma_{\min }=\sqrt{2} \Gamma_{1}^{\prime}(0)$. When $R_{0}<\hbar \Omega$ (e.g., in II-VI and III-V crystals), then IV transitions become asymmetric, i.e., they involve optical phonon absorption only. In this case transitions 2 occur to the conduction band and become real states of an interband transition, and $\Gamma_{\min }=\Gamma_{1}^{\prime}(0)$.

For excited exciton states, contrary to the ground state, an IV transition is asymmetric, because the transition 2 (with phonon emission) always occurs to the conduction band. Moreover, according to [8], the D and IV transitions become incompatible, and their total probability may exceed the classic value found for a D transition. For the $n>1$ exciton absorption band the minimal half-width always is $\Gamma_{\min }=\Gamma_{n}^{\prime}(0)$.

Taking into account the results of numerical fitting of the above model to the experimental exciton absorption spectra in InSe and GaSe crystals, we obtained the following analytical expression for $K_{n}\left(\Gamma_{n}\right)$ that gives a satisfactory description for the observed increase with temperature of the light absorption coefficient $\alpha(\hbar \omega)$ in the range of excited exciton states:

$$
\begin{aligned}
& K_{n}\left(\Gamma_{n}\right)=K_{n}^{\mathrm{D}}+K_{n}^{\mathrm{IV}}=\frac{2 K^{0}}{n^{3}} \times \\
& \times\left[\frac{1}{\sqrt{2}}+\left(1-\frac{1}{\sqrt{2}}\right) \frac{\sqrt{\Gamma_{n}^{2}-\Gamma_{n}^{\prime 2}(0)-\Gamma_{\mathrm{inh}}^{2}}}{\Gamma_{n}}\right]
\end{aligned}
$$

One can see from Fig. 1b that curve 2 (plotted from expression (5) for $\Gamma_{2}(T)$ taken according to expression (3) at $\left.\Gamma_{\min }=\Gamma_{2}^{\prime}(0)=2.81 \mathrm{meV}\right)$ satisfactorily describes the experimental $K_{2}\left(\Gamma_{2}\right)$ dependencies for InSe crystal as temperature grows, and at high temperatures the $K_{2}$ value asymptotically approaches $K_{2}{ }^{\max }=2 K^{0} / 2^{3}$. It can be seen that the initial broadening of the $n=2$ exciton absorption band and growth of its integral intensity occur at $\Gamma_{\min }=\Gamma_{2}^{\prime}(0)$. Curve 2 plotted in Fig. 1d according to expressions (5) and (3) also gives a satisfactory description for $K_{2}$ growth with temperature.

Thus, it is evident that IV transitions, along with D transitions, make a substantial contribution to formation of exciton absorption bands. One could say that the role of IV transitions becomes more important with a temperature (and number of phonons in crystal) growth. In $p$-GaSe crystals, however, contrary to $n$-InSe, in addition to the above mechanisms for exciton creation, suppression of oscillator strength for the $n=1$ exciton tran- 
Yu.I. Zhirko, I.P. Zharkov: Investigation of some mechanisms for formation of ...

sition was observed at low temperatures [2]. It manifested itself in retardation of growth of the $K_{1}\left(\Gamma_{1}\right)$ curve.

\subsection{On degeneracy of excitons with $2 D$ hole gas}

Shown in Fig. 3 (circles) are the experimental dependencies of the integral intensities $K_{1}$ (ground state) and $K_{2}$ (the first excited state) on half-width or temperature taken for pure $p$-GaSe crystal. Curves 2 present $K_{1}\left(\Gamma_{1}\right), K_{1}(T)$ and $K_{2}\left(\Gamma_{2}\right), K_{2}(T)$ dependencies for GaSe crystal. They are plotted in accordance with expressions (1) and (2) and $\Gamma_{\min }=\sqrt{2} \Gamma_{1}^{\prime}(0)=2.95 \mathrm{meV}-$ for the ground state, and expressions (3) and (5) and $\Gamma_{\min }=\Gamma_{2}^{\prime}(0)=3.81 \mathrm{meV}$ at $\Gamma_{\text {inh }}=4.2 \mathrm{meV}-$ for the $n=2$ state.
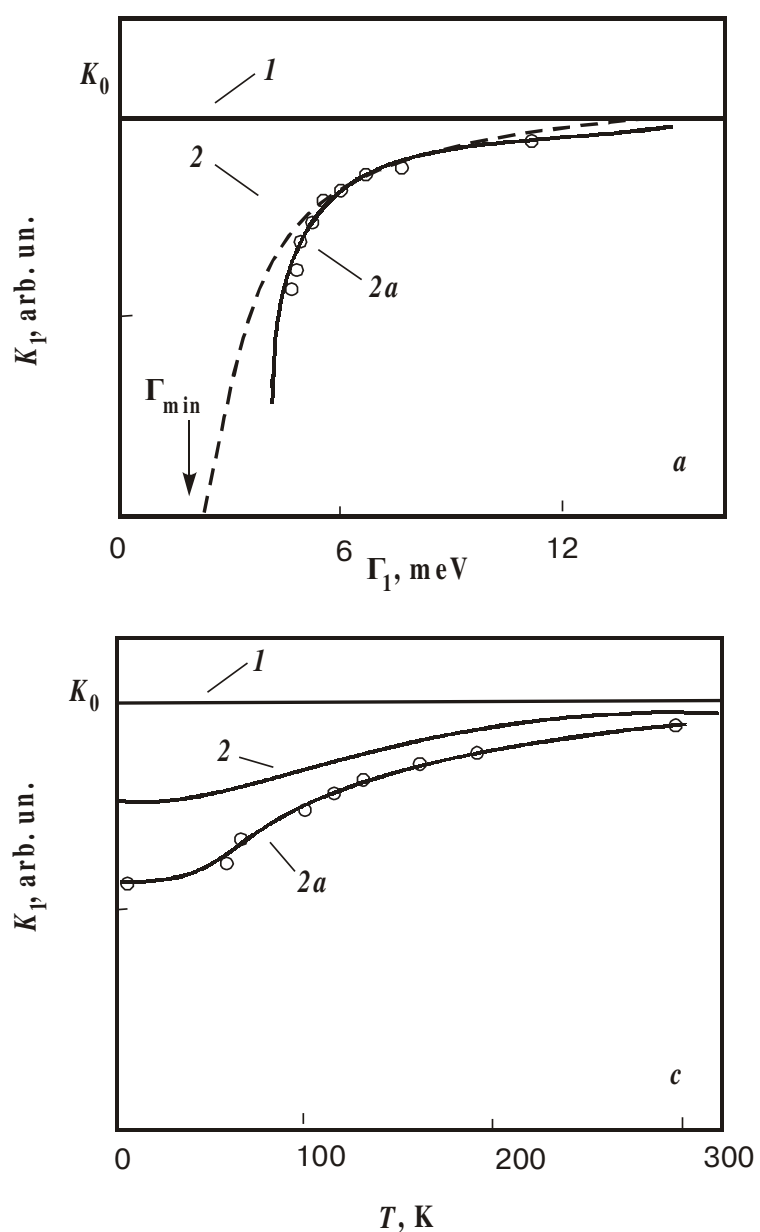

One can see from Figs $3 a$ and $3 c$ that in GaSe crystals $K_{1}$ increases somewhat slower than curve 2 ; at low temperatures this is true for $K_{1}$ dependencies on both temperature and half-width. However, as temperature grows, the experimental $K_{1}$ values are verging to curve 2 , and at big half-widths $K_{1}$ reaches its classic value, $K^{0}$. Curve 1 corresponds to the classic absorption when $K_{1}=K^{0} \equiv$ const.

According to [2], such behavior of $K_{1}\left(\Gamma_{1}\right)$ and $K_{1}(T)$ curves stems from the $n=1$ exciton state degeneracy (in the momentum space) with holes localized in 2D QWs. For $p$-GaSe, confinement of hole motion in the crystal layer planes is due to the defect stacking faults that result in appearance of potential barriers between layers. It should be noted that similar potential barriers appear also in $n$-InSe crystals. We believe, however, that in this case the barriers are not high enough to provide electron
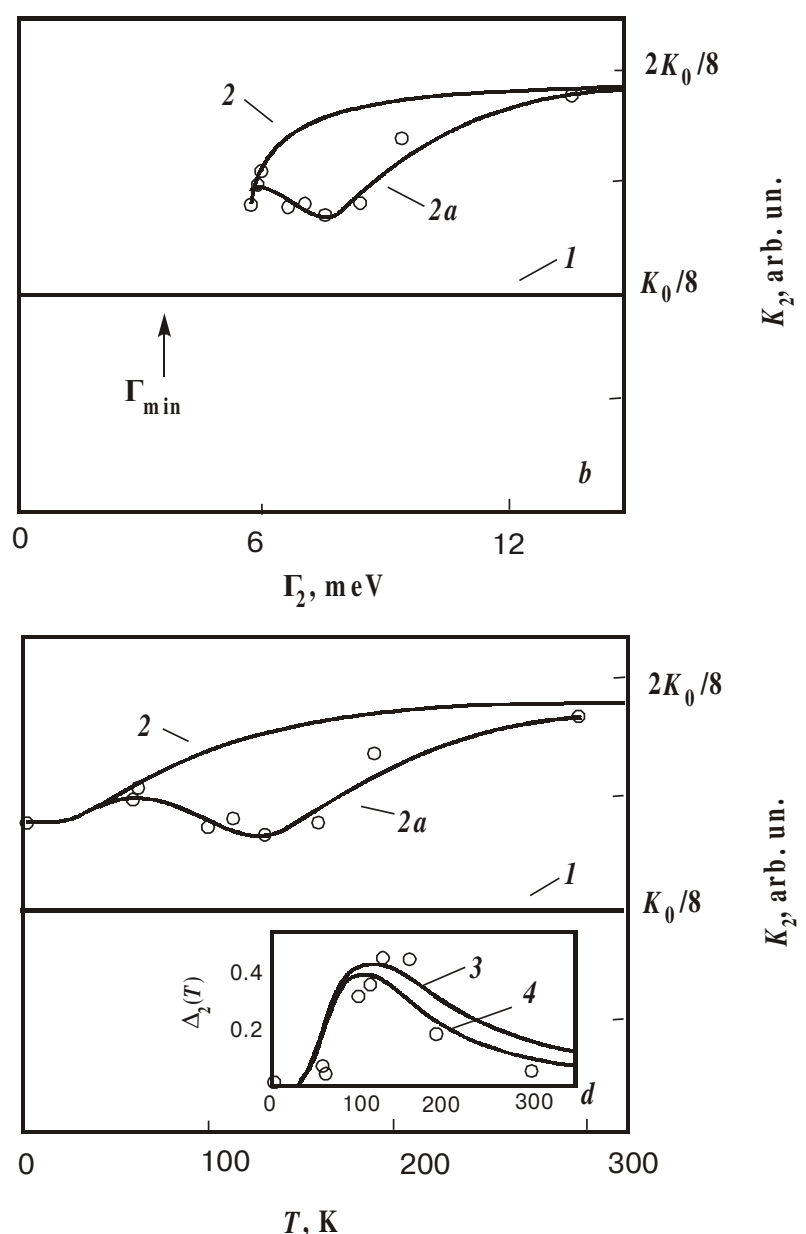

Fig. 3. $a, c-K_{1}\left(\Gamma_{1}\right)$ and $K_{1}(T)$ values for the $n=1$ exciton state in $p$-GaSe crystal at temperatures from 4.5 up to $300 \mathrm{~K}$ (circles). Curves 1 are plotted for classic absorption when $K_{1}\left(\Gamma_{1}\right)=K^{0} \equiv$ const. Curves 2 are plotted according to expression (1) with $\Gamma_{1}(T)$ according to expression (2). $b, d-K_{2}\left(\Gamma_{2}\right)$ and $K_{2}(T)$ values for the $n=2$ exciton state in $p$-GaSe crystal at temperatures from 4.5 up to $300 \mathrm{~K}$ (circles). Curves 1 are plotted for classic absorption when $K_{2}\left(\Gamma_{2}\right)=K^{0} / 8$. Curves 2 are plotted according to expressions (5) at $\Gamma_{\text {inh }}=4.2 \mathrm{meV}$ with $\Gamma_{2}(T)$ according to expression (3). Curves $2 a$ are the spline lines for experimental data. Insert of Fig. $3 \mathrm{~d}: \Delta_{2}(T)$ values for the $n=2$ exciton state in $p$-GaSe crystal at temperatures from 4.5 up to $300 \mathrm{~K}$ (circles). Curve 3 is plotted according to expression (6). Curve 4 is plotted with the account of evaporation of localized hole gas. 
Yu.I. Zhirko, I.P. Zharkov: Investigation of some mechanisms for formation of ...

localization, because in $n$-InSe $m_{\mathrm{e}}^{\perp} / m_{\mathrm{e}} \| \cong 1$ (here the indices $\|$ and $\perp$ refer to directions along the layers and normal to them, respectively), and electrons demonstrate $3 \mathrm{D}$ behavior. Nevertheless, some authors [12] think that $n$-InSe doping (say, with $\mathrm{Zn}$ ) results in appearance of additional 2D electrons (of low concentrations) whose mobility is low.

Contrary to the above, in $p$-GaSe crystals, due to a marked hole effective mass anisotropy $\left(m_{\mathrm{h}}^{\perp} / m_{\mathrm{h}} \|=4-5\right)$, localization of hole motion in the layer planes occurs in the presence of the crystal layer stacking faults. As temperature grows, the holes localized in QWs are redistributed to the states of higher energies. This leads to lifting off degeneracy in the momentum space between the excitons and holes. As a result, both the polariton mechanism and oscillator strength of exciton transition are restored. The results obtained in [2] for the $n=1$ exciton state are in a good agreement with a model (advanced by F.G. Pikus [13]) for exciton behavior in QWs with 2D electron (hole) gas. For $n$-InSe and $p$-GaSe crystals, however, excitons (contrary to holes) remain 3D behavior. At low temperatures the absorption spectra in both crystals demonstrate a well-pronounced $3 \mathrm{D}$ exciton series $E_{n}=$ $=E_{g}-R_{0} / n^{2}$.

At the same time, the photoluminescence spectra of these crystals [14-16] at low temperatures have a fine structure in the region of emission of the ground exciton state. This fine structure is due to weak exciton localization at stacking faults. Moreover, as it was shown in [14], it is just the stacking faults in $n$-InSe that lead to mixing of two close $\varepsilon$ - and $\gamma$-modifications, thus finally resulting in appearance of potential barriers between layers. It was shown ibidem that such regions are situated in a quite nonuniform way; their areas in a plane lie, as a rule, between 0.2 and $2 \mathrm{~mm}^{2}$.

For GaSe crystal the experimentally determined integral intensity of the $n=2$ exciton band as a function of a half-width and temperature is shown in Figs $3 \mathrm{~b}$ and $3 \mathrm{~d}$ (circles). In this crystal (as for InSe) the inequality $K_{2}>K^{0} / 2^{3}$ holds, and $K_{2}$ value increases with the crystal temperature. In this case, however, the experimental $K_{2}\left(\Gamma_{2}\right)$ and $K_{2}(T)$ curves are non-monotonic and differ substantially from the curve 2 that describes $K_{2}$ behavior as temperature grows, both D and IV transitions being taken into account. One can see that at moderate $(4.5-60 \mathrm{~K})$ temperatures the experimental $K_{2}\left(\Gamma_{2}\right)$ and $K_{2}(T)$ curves for GaSe practically coincide with the curve 2 . At further temperature rise, however, the integral intensity at first decreases, but then, at higher $(>130 \mathrm{~K})$ temperatures, its experimental values are gradually approaching the curve 2 .

The obtained experimental results serve as direct verification of the assumption (stated earlier in [2]) that in $p$ GaSe crystals degeneracy in the momentum space exists between excitons and holes localized in QWs. At first, such degeneracy leads to suppression of oscillator strength for the $n=1$ exciton transition. Temperature growth results in increasing the mean kinetic energy of hole gas. This displays itself in redistribution of holes to the states of higher energies, thus restoring the polariton mecha- nism for the ground exciton state. The above hole redistribution, in its turn, leads to degeneracy of holes and excited exciton states. This shows itself in the fact that, at some temperatures, the integral intensity of absorption bands of excited exciton states goes down. Further hole redistribution to the states of higher energies with temperature results in integral intensity restoration for the $n=2,3 \ldots$ exciton absorption bands. Indeed, similar non-monotonic behavior is also observed for the $n=3$ exciton absorption band in GaSe, but at a higher temperature.

Shown in Fig. $3 \mathrm{~d}$ (insert) is the quantity $\Delta_{2}(T)=K_{2}(T)-$ $-K_{2} \exp (T)$ for the $n=2$ exciton absorption band in GaSe (circles). This quantity characterizes temperature dependence of the departure of experimentally found $K_{2}$ value $\left(K_{2}{ }^{\text {exp }}\right.$ ) from the $K_{2}(T)$ function presented by curve 2 (Fig. $3 \mathrm{~d})$. It should be noted that, despite the fact that in this case one deals with non-monotonic behavior of the integral intensities of exciton absorption bands in crystals with holes localized in QWs, the experimental results for $\Delta_{2}(T)$ rather satisfactorily reflect the Maxwellian temperature distribution for hole gas:

$$
F_{n}(E, T)=\left(E_{F}-E_{n}\right) \frac{d}{d T} f_{n}(E, T) .
$$

Here $f_{n}(E, T)=1 /\left(e^{\left[E_{F}-E_{n}(T)\right] / k_{\mathrm{B}} T}+1\right)$ is the Fermi function for the degenerated hole gas in QW; $E_{n}$ and $E_{F}$ are, respectively, the energy of the $n=1,2,3 \ldots$ exciton state and Fermi level for holes localized in QWs. Curve 3 plotted according to expression (6) obeys the $\Delta_{2}(T)$ dependence at the low-temperature slope only, and considerably departs from the experimental dependence at the high-temperature slope. We believe that this departure is caused by "evaporation" of some amount of hole gas as temperature grows; some portion of holes (localized in QWs) with increased kinetic energy overcome the potential barriers and become 3D. One can see that the curve 4 plotted with allowance made for "evaporation" gives a considerably better description for $K_{n}(T)$ behavior in the case when exciton states are degenerate with the hole gas localized in QWs.

We would like to note that similar conclusions on a non-monotonic $K_{n}\left(\Gamma_{n}\right)$ growth as temperature increases from 4.5 up to $300 \mathrm{~K}$ have been made by us also for $\mathrm{GaSe}$ crystals doped and intercalated with Mn.

The effects of exciton-hole interaction [17] are known to lead, for instance, to appearance of trion states (bound exciton-electron/hole states in structures with QWs containing 2D electron/hole gas) in the $\mathrm{ZnSe} / \mathrm{ZnMnSSe}-$ based structures with QWs. The results of our investigations of some mechanisms for creation and decay of excitons in layered InSe and GaSe crystals enable one to conclude that, despite the fact that in $p$-GaSe crystals excitons are degenerate in the momentum space with $2 \mathrm{D}$ hole gas, the above effects seem not to occur. This is due, firstly, to the fact that in our case Wannier excitons do not lower their dimensionality and remain practically $3 \mathrm{D}$, so the binding energy of the above trion complexes 
would be very low in the 3D case. Secondly, the holes in a 2D QW cannot manage to orient their spins (and form by that a considerable number of $X^{+}$-trions) during the time when a 3D exciton is near this $2 \mathrm{D} \mathrm{QW}$ with holes.

\section{Conclusions}

Our experimental investigations of the absorption spectra in InSe and GaSe crystals performed over a wide temperature range, together with spectra calculations made within the framework of the classic Elliott-Toyozawa model (using various models for exciton creation, radiation and decay), made it possible:

- to show that growth of absorption band integral intensities $K_{1}$ (ground exciton state ) and $K_{n}$ (excited exciton states) with temperature proceeds with involvement of both D (photon $\rightarrow$ exciton $\rightarrow$ photon, at $\boldsymbol{k}=0$ ) and IV (photon \pm phonon $\rightarrow$ exciton $\rightarrow$ photon \pm phonon, at $\boldsymbol{k} \cong 0$ ) optical transitions, the latter occurring with optical phonon creation/annihilation;

- to show that for the $n=1$ exciton state the above D and IV transitions are compatible. Depending on the difference between the exciton and phonon binding energies, the IV transitions to the $n=1$ exciton state may be either symmetric (with phonon creation/annihilation), or asymmetric (with phonon annihilation only). For symmetric IV transitions $\Gamma_{\min }=\sqrt{2} \Gamma_{1}^{\prime}(0)$, while for asymmetric ones $\Gamma_{\min }=\Gamma_{1}^{\prime}(0)$;

- to determine that D and IV transitions become incompatible for excited exciton states. Therefore the integral intensity of absorption bands for excited exciton states, $K_{n}$, exceeds $K^{0} / n^{3}$ (here $K^{0}$ is the classic value of integral intensity for the $n=1$ exciton absorption band) and grows with temperature. At high temperatures $K_{n} \rightarrow$ $2 K^{0} / n^{3}$;

- to find that presence of $2 \mathrm{D}$ gas of charge carriers (electrons/holes localized in QWs) degenerate in the momentum space with the exciton states results in suppression of the oscillator strength for exciton transition (for the ground, as well as excited, states);

- to experimentally demonstrate that temperature increase results in hole redistribution to the states of higher energies. This appears as consecutive (from the ground exciton state to excited ones) suppression and restoration of the integral characteristics of the exciton absorption bands.

\section{Acknowledgements}

This work was partially supported by the Basic Research Fund of Ukraine (Project No $\Phi 7 / 310-2001$ ).

\section{References}

1. Yu.I. Zhirko, Investigation of the light absorption mechanisms near exciton resonance in layered crystals. $N=1$ state exciton absorption in InSe // phys. stat. sol. (b) 213, pp. 93106 (1999).

2. Yu.I. Zhirko, Investigation of the light absorption mechanisms near exciton resonance in layered crystals. Part 2. $N=$ 1 state exciton absorption in GaSe // phys. stat. sol. (b) 219 , pp. 47-61 (2000)

3. A.S. Davydov, A.A. Serikov, Quantum-statistical theory of light propagation in crystals // phys. stat. sol. (b). 56, pp. 351363 (1973).

4. L.N. Kurbatov, A.I. Dirochka, V.A. Sosin, Effect of exciton polaritons on the GaTe absorption edge. I. Temperature dependence of absorption // Fiz. Tekh. Poluprov. 13(1), pp. 7582 (1979) (in Russian); L.N. Kurbatov, A.I. Dirochka, V.A. Sosin, Effect of exciton polaritons on the GaTe absorption edge. II. Dependence of absorption on sample thickness // Fiz. Tekh. Poluprov. 13(1), pp. 83-88 (1979) (in Russian).

5. A. Bosacchi, B. Bosacchi, S. Franchi, Polariton Effects in the Exciton Absorption of GaSe // Phys. Rev. Lett., 36(18), pp. 1096-1086 (1976).

6. I.V. Blonsky, B.M. Nitsovich, A.S. Krochuk, A.V. Franiv, Effect of indirect phototransitions on the temperature dependence of exciton absorption band intensity in InSe // $U \mathrm{kr}$. Fiz. Zhurn. 29(1), pp. 131-132, (1984) (in Russian).

7. A.S. Davydov, Solid State Theory, Nauka, Moscow (1976) (in Russian).

8. Yu.I. Zhirko, I.P. Zharkov, Light absorption by excited exciton states in layered InSe crystals // Semiconductor Physics, Quantum Electronics \& Optoelectronics 5(2), pp. 156-162 (2002).

9. R. Le Toullec, N. Piccioli, J.C. Chervin, Optical properties of the band-edge exciton in GaSe crystals at $10 \mathrm{~K} / /$ Phys. Rev. B 22, pp. 6162-6170 (1980).

10. B.M. Nitsovich, V.V. Nitsovich, V.D. Falenchuk, Integral intensity of exciton absorption band // Fiz. Tverd. Tela 19, pp. 3438-3439 (1977) (in Russian); B.M. Nitsovich, V.V. Nitsovich, G.M. Pestryakov, V.D. Falenchuk, Indirect exciton phototransition in layered semiconductors // Fiz. Tverd. Tela 27, pp. 1881-1883 (1985) (in Russian).

11. M.S. Brodin, I.V. Blonsky, B.M. Nitsovich, V.V. Nitsovich, Dynamic Effects in Multicomponent Gas of Quasiparticles, Naukova Dumka, Kiev (1990) (in Russian).

12. A. Segura, B. Mari, J. Martinez-Pastor, A. Chevy, Three dimensional electrons and two-dimensional electric subbands in the transport properties of tin-doped $n$-type indium selenide: Polar and homopolar phonon scattering // Phys. Rev. B 43(6), pp. $4953-4965$ (1991).

13. F.G. Pikus, Excitons in quantum wells with $2 \mathrm{D}$ electron gas // Fiz. Tverd. Tela 26, pp. 45-58, (1992) (in Russian).

14. Yu.P. Gnatenko, Yu.I. Zhirko, Z.D. Kovalyuk, V.M. Kaminski, The features of exciton luminescence spectra of $g$-InSe crystals with stacking faults // Fiz. Tverd. Tela, 28(12), pp. 3591-3594 (1986) (in Russian).

15. J.J. Forney, K. Maschke, E. Moozer, Fine-structure splitting of the ground state excitons in layered materials with stacking faults // Nuovo Cim. 38B(2), pp. 418-422 (1977).

16. N. Kuroda, Y. Nishina, Stacking-fault splitting of exciton spectrum in GaSe // Nuovo Cim. 32B(1), pp. 109-115 (1976).

17. V.P. Kochereshko, R.A. Suris, D.R. Yakovlev, Effects of exciton-electron interaction in the structures with quantum wells containing 2D electron gas // Uspekhi Fiz. Nauk, 170(3), pp. 335-338 (2000) (in Russian). 